\title{
Substituent effects on the relative rates and free energies of ortho- lithiation reactions: families of fluorobenzenes as the substrates
}

\author{
Florence Mongin, ${ }^{* a}$ Christophe Curty, ${ }^{\mathrm{a}}$ Elena Marzi, ${ }^{\mathrm{b}}$ Frédéric R. Leroux, ${ }^{\mathrm{c}}$ \\ and Manfred Schlosser*ab \\ ${ }^{a}$ Institut de Chimie organique de l'Université, Bâtiment de chimie, 1015 Lausanne, Switzerland \\ ${ }^{b}$ Institute for Chemical Sciences and Engineering, Bâtiment de chimie, Ecole Polytechnique \\ Fédérale (EPFL), 1015 Lausanne, Switzerland \\ ${ }^{c}$ Laboratoire de Chimie Moléculaire, UMR CNRS 7509, Université de Strasbourg, ECPM, 25 \\ Rue Becquerel, 67087 Strasbourg, France \\ E-mail: florence.mongin@univ-rennes1.fr
}

Dedicated to Professor Manfred Schlosser in honor of his scientific achievements

DOI: http://dx.doi.org/10.3998/ark.5550190.p008.926

\begin{abstract}
2-, 3- and 4-substituted fluorobenzenes and 5-substituted 1,3-difluorobenzenes were metalated with sec-butyllithium (LIS) and with lithium 2,2,6,6-tetramethylpiperidide (LiTMP) under irreversible conditions in order to determine the rates of reaction relative to the unsubstituted parent compounds (fluorobenzene and 1,3-difluorobenzene). In addition, the pairs of resulting aryllithiums were subjected to acid-base equilibration to furnish the thermodynamic stabilities (or: basicities) of these species again relative to the parent compounds. Not surprisingly, the effect diminishes with the distance of a given substituent to the lithiation center (ortho > meta > para) and it reaches its maximum at the ground state equilibration of the organometallic intermediate whereas it fades away at transition states, in particular reactant-like ones. Fluorine, the most powerful activator in the entire series if located at an ortho position, increases the rates of LIS- and LiTMP-promoted metalations by respectively 2 and 3 powers of ten, but by 7 to 8 powers of ten the aryllithium equilibrium stability.
\end{abstract}

Keywords: Basicity, fluoroarenes, metalation reactions, relative rate measurements, substituent effects 


\section{Introduction}

In an attempt to popularize his pioneering work in the organolithium area, G. Wittig wanted to link it as a counterpart to the already well established "Cationo-Chemie" of A. v. Meerwein and thus coined the term "Carbaniono-Chemie". ${ }^{1-3}$ However, the new label implies an oversimplification. Unlike carbocations, free carbanions can in general, apart from a handful of peculiar exceptions, not exist in solution. ${ }^{4}$ As a consequence, the same organic derivatives of various alkali and alkali-earth metals are by no means different incarnations of an identical carbanion but each of them is a distinct chemical entity. Therfore, all of them exhibit individual reactivity and selectivity profiles. ${ }^{4}$

To save the carbanion concept, one may consider organometallic species as a blend between unpolar and polar structures and attribute "carbanion characters" as a function of the metal involved. Thus, one could assume organo-magnesium, -lithium, -sodium, -potassium and cesium compounds to mirror, respectively, $20 \%, 40 \%, 60 \%, 80 \%$ and $90 \%$ of the properties of the corresponding free carbanion. ${ }^{4}$ Unfortunately, the postulated proportionality is unable to account for metal-specific reactivity and stability patterns. For example, phenylmagnesium bromide adds quantitatively onto the carbonyl double bond of acetophenone whereas phenylpotassium generates exclusively the enolate by $\alpha$-proton abstraction. ${ }^{5}$ The free protonation energies of the ethyl and isopropyl anions in the gas phase exceed that of the methyl anion reference by $2.8 \mathrm{kcal} \mathrm{mol}^{-1}$ and the tert-butyl anion falls behind by $2.9 \mathrm{kcal} \mathrm{mol}^{-16}$ whereas the basicity monotonously increases in the series methyllithium, ethyllithium (or butyllithium), isopropyllithium (or sec-butyllithium) and tert-butyllithium. ${ }^{7,8}$ Finally, heterosubstituent effects on the proton affinities of "naked" carbanions and the corresponding lithiated species may differ strikingly. The 1-naphthyl anion and its 8-methoxy and 8-fluoro congeners have practically the same basicity whereas 8-methoxy-1-naphthyllithium and 8-fluoro-1-naphthyllithium are, respectively, 9 and $10 \mathrm{kcal} \mathrm{mol}^{-1}$ more stable (i.e., less basic) than 1-naphthyllithium itself. ${ }^{9}$

In our opinion, it is of fundamental importance to provide a rational basis for the understanding of phenomena as those evoked above. In particular, one should strive to find out how a given carbon skeleton and attached substituents can stabilize excess negative charge as present in carbanions and organometallic derivatives thereof. However, it does not suffice to monitor just such ground state species. The investigation should be extended to the ease of their generation and transformation. In other words, carbanions and organometallics should be also probed as imaginary constituents of transition states.

The resulting knowledge will prove valuable in predicting the outcome of stoichiometric or catalytic organometallic reactions. Moreover, it will lead to a deeper insight into the nature of electronic effects, their attenuation and transmission. This has always been one of the most fascinating topics of physical organic chemistry.

While pursuing these objectives, the present study restricts itself to heterosubstituted fluorobenzenes as model substrates. In this area, abundant data have been collected about the thermodynamic stability of the free anions by gas phase experiments, ${ }^{10-12}$ computational efforts ${ }^{13}$ 
and base-catalyzed isotope exchange. ${ }^{14-18}$ Therefore, we could focus in the following on the assessment of metalation rates and organometallic basicity.

\section{Results}

The first step was to select appropriate model substrates. As we have experienced previously, ${ }^{19}$ monosubstituted benzenes have considerable shortcomings as substrates for kinetic measurements. Several of them (chlorobenzene, anisole, benzotrifluoride) do not react at all with lithium 2,2,6,6-tetramethylpiperidide (LiTMP) under standard conditions. The other one of our two standard reagents, sec-butyllithium (LIS), attacks the parent compound benzene with extreme sluggishness and the gap between benzene and the least reactive derivative thereof, benzotrifluoride, is too big to make the relative rate measurement reliable. Moreover, monosubstituted benzenes, except benzotrifluoride, ${ }^{20,21}$ undergo metalation exclusively at an ortho position and thus do not offer any information about the proton mobility at meta and para positions.

Our choice of the model substrates 1-4 (Scheme 1) made it possible to probe the influence of substituents $\mathbf{X}$ accommodated at ortho, meta and para positions with respect to the lithiated center. Our set of substituents (Scheme 1) comprised trimethylsilyl, methoxy, the halogens fluorine, chlorine, bromine, and iodine and trifluoromethyl. Fluorine being the most powerful ortho-directing neighboring group in this series of simple, non-functional substituents, rapid and clean metalation was secured. Last but not least, the data collected should mirror the genuine contribution of each individual substituent $\mathbf{X}$ as fluorine effects on the kinetic ${ }^{12}$ and thermodynamic ${ }^{13}$ acidity of arenes are additive to those of other fluorine atoms and presumably also to those of most, if not all, other substituents.

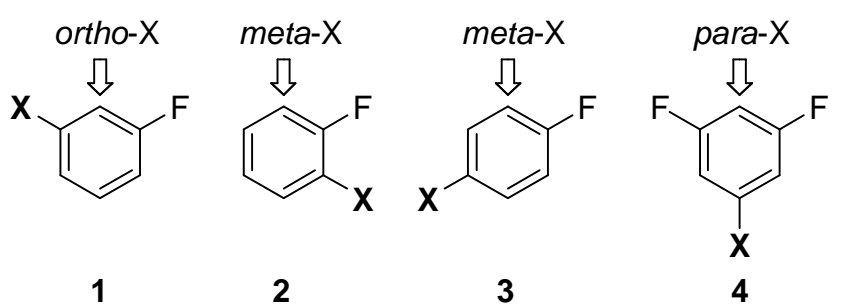

Scheme 1. Model substrates 1-4 (a: $X=H ; \mathbf{b}: X=S_{1 M e} ; \mathbf{c}: X=O M e ; \mathbf{d}: X=F ; \mathbf{e}: X=C l ; f: X$ $\left.=\mathrm{Br} ; \mathbf{g}: \mathrm{X}=\mathrm{I} ; \mathbf{h}: \mathrm{X}=\mathrm{CF}_{3}\right)$ for the assessment of relative metalation rates $\left(k_{\text {rel }}\right)$ and metalation equilibria $\left(K_{\text {rel }}\right)$.

Before measuring rates and equilibria, we had to perform a number of control experiments. First, we checked the efficacy of our quenching methods. The trapping of 2-fluorophenyllithium and other intermediates with the two electrophiles employed, chlorotrimethylsilane and carbon dioxide, proved to be complete in less than 30 seconds. Next we tested to what extent transmetalation between an organolithium intermediate and an unconsumed model substrate 
might occur under the given operational conditions. Previously, we observed almost no change due to transmetalation when exposing 1,2- and 1,4-difluorobenzene to the action of 2fluorophenyllithium at $-75{ }^{\circ} \mathrm{C}$ during $2 \mathrm{~h}$. In contrast, significant amounts of 1,2,4trifluorobenzene were converted by 2,6-difluorophenyllithium into 2,3,6-trifluorophenyllithium (36\%) and pentafluorobenzene by 2,3,5,6-tetrafluorophenyllithium into pentafluorophenyllithium $(58 \%)$ at $0.5 \mathrm{M}$ concentrations. ${ }^{22}$ In the present context, we have followed the transmetalation between 1,4-difluorobenzene and 2-fluoro-5-chlorophenyllithium and between 1-chloro-4-fluorobenzene and 2,5-difluorophenyllithium over a period of 820 hours without attaining equilibrium (which would require estimated 2000 hours). The exposure time of all substrate pairs to LIS have been limited to 15 minutes, the initial composition of the two metalated intermediates should be only negligibly $(<5 \%)$ affected by subsequent transmetalation. The scenario changed completely when 2-fluoro-5-chlorophenyllithium and 2,5difluorophenyllithium were generated by LiTMP in the presence of excess arene precursors. Incessant reprotonation by the 2,2,6,6-tetramethylpiperidine set free and repeated deprotonation by LiTMP caused the 2-fluoro-5-chlorophenyllithium/2,5-difluorophenyllithium ratio to drift from the initial 56:44 in the course of 45 minutes to the final 72:28 composition. Remarkably enough, the equilibration process could be rigorously prevented if the LiTMP-promoted deprotonation was accomplished under "in situ trapping" conditions, ${ }^{23-29}$ i.e. in the presence of excess chlorotrimethylsilane.

Guided by this exploratory work, the substrates 1 (Table 1), 2 (Table 2), 3 (Table 3) and $\mathbf{4}$ (Table 4) were subjected to competition kinetic measurements using LIS and LiTMP under "in situ trapping" conditions and to acid-base equilibration using LiTMP during prolonged reaction times in tetrahydrofuran (THF) at $-75^{\circ} \mathrm{C}$. The data thus gathered were found to be reproducible within narrow limits of error $(<10 \%)$.

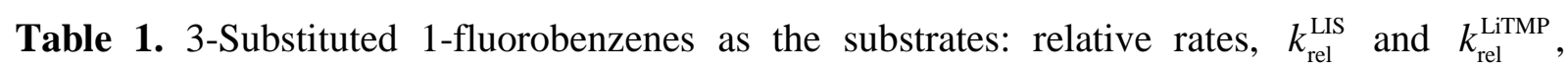
respectively, upon reaction with LIS and with LiTMP (by applying the "in situ trapping" protocol) and relative equilibrium constants $K_{\text {rel }}$ (by applying the "LiTMP-incubation" protocol) ${ }^{\mathrm{a}}$

\begin{tabular}{llcll}
\hline Substrate & $\mathrm{X}$ & $k_{\text {rel }}^{\text {LIS }}$ & $k_{\text {rel }}^{\text {LiTMP }}$ & $K_{\text {rel }}$ \\
\hline $\mathbf{1 a}$ & $\mathrm{H}$ & $1.0 \times 10^{0}$ & $1.0 \times 10^{0}$ & $1.0 \times 10^{0}$ \\
$\mathbf{1 b}$ & $\mathrm{SiMe}_{3}$ & $-^{-}$ & $-\mathrm{b}$ & $-^{\mathrm{b}}$ \\
$\mathbf{1 c}$ & $\mathrm{OMe}$ & $-^{\mathrm{c}}$ & $1.5 \times 10^{2}$ & $1.0 \times 10^{1}$ \\
$\mathbf{1 d}$ & $\mathrm{F}$ & $1.1 \times 10^{2}$ & $1.4 \times 10^{3}$ & $1.3 \times 10^{8}$ \\
$\mathbf{1 e}$ & $\mathrm{Cl}$ & $1.0 \times 10^{2}$ & $1.1 \times 10^{3}$ & $1.0 \times 10^{7}$ \\
$\mathbf{1 f}$ & $\mathrm{Br}$ & $-^{-}$ & $7.4 \times 10^{2}$ & $1.2 \times 10^{7}$ \\
$\mathbf{1 g}$ & $\mathrm{I}$ & $-^{-}$ & $7.2 \times 10^{2}$ & $1.2 \times 10^{7}$ \\
$\mathbf{1 h}$ & $\mathrm{CF}_{3}$ & $5.0 \times 10^{1}$ & $1.8 \times 10^{2}$ & $5.8 \times 10^{5}$ \\
\hline
\end{tabular}

${ }^{a}$ All rate and equilibrium constants are statistically corrected for the number of equivalent acidic sites. ${ }^{\mathrm{b}}$ Unavailable (see text and Scheme 2). ${ }^{\mathrm{c}}$ Not performed. 
Bromo- and iodoarenes metalation rates could of course not be assessed with LIS as no hydrogen/metal but only halogen/metal permutation would have taken place. The corresponding relative rates of 2- and 4-fluoroanisole are equally missing as LIS does not attack selectively the fluorine adjacent site. ${ }^{30}$ Finally, (3-fluorophenyl)trimethylsilane had to be omitted in both the LIS and LiTMP experiments as the metalation occurred at the 4- rather than at the 2-position. ${ }^{31}$ The same behavior was observed in the case of 3-fluoro- $N, N$-dimethylaniline. ${ }^{32-33}$ Otherwise, we would have included this compound into our set of model substrates together with 2-fluoro- and 4-fluoro- $N, N$-dimethylaniline which both underwent the LIS-mediated metalation selectively, next to the fluorine-bearing site, and efficaciously.<smiles>COc1c(F)cccc1C(=O)F</smiles>

2c<smiles>COc1ccc(F)c(F)c1</smiles>

$3 c$

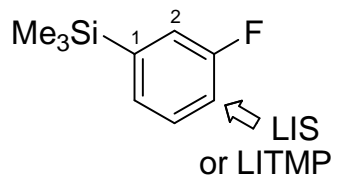

1b

Scheme 2. No regioselective attack of LIS at the 3-position of 2- and 4-fluoroanisole, no attack of LIS or LiTMP at the 2-position of 3-(fluorophenyl)trimethylsilane and 3-fluoro- $N, N$ dimethylaniline.

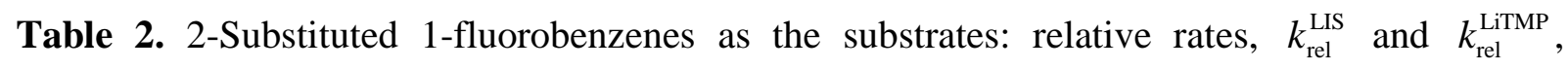
respectively, upon reaction with LIS and with LiTMP (by applying the "in situ trapping" protocol) and relative equilibrium constants $K_{\text {rel }}$ (by applying the "LiTMP-incubation" protocol) ${ }^{\mathrm{a}}$

\begin{tabular}{llcll}
\hline Substrate & $\mathrm{X}$ & $k_{\text {rel }}^{\text {LIS }}$ & $k_{\text {rel }}^{\text {LiTMP }}$ & $K_{\text {rel }}$ \\
\hline $\mathbf{2 a}$ & $\mathrm{H}$ & $1.0 \times 10^{0}$ & $1.0 \times 10^{0}$ & $1.0 \times 10^{0}$ \\
$\mathbf{2 b}$ & $\mathrm{SiMe}_{3}$ & $4.8 \times 10^{-1}$ & $3.9 \times 10^{-1}$ & $2.2 \times 10^{-1}$ \\
$\mathbf{2 c}$ & $\mathrm{OMe}$ & $-\mathrm{b}$ & $2.9 \times 10^{0}$ & $3.2 \times 10^{0}$ \\
$\mathbf{2 d}$ & $\mathrm{F}$ & $2.0 \times 10^{1}$ & $5.0 \times 10^{1}$ & $2.5 \times 10^{2}$ \\
$\mathbf{2 e}$ & $\mathrm{Cl}$ & $4.0 \times 10^{1}$ & $1.0 \times 10^{2}$ & $5.2 \times 10^{2}$ \\
$\mathbf{2 f}$ & $\mathrm{Br}$ & $-\mathrm{b}$ & $1.4 \times 10^{2}$ & $9.6 \times 10^{2}$ \\
$\mathbf{2 g}$ & $\mathrm{I}$ & $-\mathrm{b}$ & $1.1 \times 10^{2}$ & $4.0 \times 10^{2}$ \\
$\mathbf{2 h}$ & $\mathrm{CF}_{3}$ & $6.0 \times 10^{1}$ & $1.9 \times 10^{2}$ & $3.8 \times 10^{3}$ \\
\hline
\end{tabular}

${ }^{a}$ All rate and equilibrium constants are statistically corrected for the number of equivalent acidic sites. ${ }^{\mathrm{b}}$ Unavailable (see text and Scheme 2). 


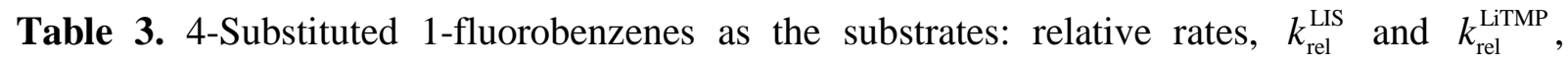
respectively, upon reaction with LIS and with LiTMP (by applying the "in situ trapping" protocol) and relative equilibrium constants $K_{\text {rel }}$ (by applying the "LiTMP-incubation" protocol) ${ }^{\mathrm{a}}$

\begin{tabular}{llcll}
\hline Substrate & $\mathrm{X}$ & $k_{\text {rel }}^{\text {LI }}$ & $k_{\text {rel }}^{\text {LiTM }}$ & $K_{\text {rel }}$ \\
\hline 3a & $\mathrm{H}$ & $1.0 \times 10^{0}$ & $1.0 \times 10^{0}$ & $1.0 \times 10^{0}$ \\
$\mathbf{3 b}$ & $\mathrm{SiMe}_{3}$ & $7.5 \times 10^{-1}$ & $3.3 \times 10^{-1}$ & $2.8 \times 10^{-1}$ \\
$\mathbf{3 c}$ & $\mathrm{OMe}$ & $-^{\mathrm{b}}$ & $3.1 \times 10^{0}$ & $2.1 \times 10^{0}$ \\
$\mathbf{3 d}$ & $\mathrm{F}$ & $1.0 \times 10^{1}$ & $4.8 \times 10^{1}$ & $1.3 \times 10^{2}$ \\
$\mathbf{3 e}$ & $\mathrm{Cl}$ & $3.0 \times 10^{1}$ & $1.6 \times 10^{2}$ & $1.1 \times 10^{3}$ \\
$\mathbf{3 f}$ & $\mathrm{Br}$ & $-\mathrm{b}$ & $3.5 \times 10^{2}$ & $2.0 \times 10^{3}$ \\
$\mathbf{3 g}$ & $\mathrm{I}$ & $-^{\mathrm{b}}$ & $3.2 \times 10^{2}$ & $1.8 \times 10^{3}$ \\
$\mathbf{3 h}$ & $\mathrm{CF}_{3}$ & $3.5 \times 10^{1}$ & $1.3 \times 10^{2}$ & $3.4 \times 10^{3}$ \\
\hline
\end{tabular}

${ }^{a}$ All rate and equilibrium constants are statistically corrected for the number of equivalent acidic sites. ${ }^{\mathrm{b}}$ Unavailable (see text and Scheme 2).

Table 4. 5-Substituted 1,3-difluorobenzenes as the substrates: relative rates, $k_{\text {rel }}^{\text {LIS }}$ and $k_{\text {rel }}^{\text {LiTMP }}$, respectively, upon reaction with LIS and with LiTMP (by applying the "in situ trapping" protocol) and relative equilibrium constants $K_{\text {rel }}$ (by applying the "LiTMP-incubation" protocol) ${ }^{\mathrm{a}}$

\begin{tabular}{llcll}
\hline Substrate & $\mathrm{X}$ & $k_{\text {rel }}^{\text {LIS }}$ & $k_{\text {rel }}^{\text {LiTMP }}$ & $K_{\text {rel }}$ \\
\hline $\mathbf{4 a}$ & $\mathrm{H}^{\mathrm{b}}$ & $1.0 \times 10^{0}$ & $1.0 \times 10^{0}$ & $1.0 \times 10^{0}$ \\
$\mathbf{4 b}$ & $\mathrm{SiMe}_{3}$ & $9.1 \times 10^{-1}$ & $9.7 \times 10^{-1}$ & $6.8 \times 10^{-1}$ \\
$\mathbf{4 c}$ & $\mathrm{OMe}$ & $-^{\mathrm{c}}$ & $7.1 \times 10^{-1}$ & $3.0 \times 10^{-1}$ \\
$\mathbf{4 d}$ & $\mathrm{F}$ & $4.8 \times 10^{-1}$ & $1.0 \times 10^{0}$ & $3.3 \times 10^{0}$ \\
$\mathbf{4 e}$ & $\mathrm{Cl}$ & $1.5 \times 10^{0}$ & $3.3 \times 10^{0}$ & $2.7 \times 10^{1}$ \\
$\mathbf{4 f}$ & $\mathrm{Br}$ & ${ }^{\mathrm{d}}$ & $3.1 \times 10^{0}$ & $2.0 \times 10^{1}$ \\
$\mathbf{4 g}$ & $\mathrm{I}$ & ${ }^{\mathrm{d}}$ & $2.7 \times 10^{0}$ & $1.5 \times 10^{1}$ \\
$\mathbf{4 h}$ & $\mathrm{CF}_{3}$ & ${ }^{-\mathrm{c}}$ & $-^{\mathrm{c}}$ & $-^{\mathrm{c}}$ \\
\hline
\end{tabular}

${ }^{\text {a }}$ All rate and equilibrium constants are statistically corrected for the number of equivalent acidic sites. ${ }^{\mathrm{b}}$ Relative to fluorobenzene (1a): $1.0 \times 10^{1}, 4.8 \times 10^{1}$ and $1.3 \times 10^{2}$, respectively (see Table 1). ${ }^{\mathrm{c}}$ Not performed. ${ }^{\mathrm{d}}$ Unavailable (see text).

\section{Discussion}

To compile temperature-invariant substituent effects, the relative rate and equilibrium constants were converted into free activation energy and thermodynamic stability differences $\Delta \Delta \mathrm{G}^{\dagger}$ and 
$\Delta \mathrm{G}^{\circ}$, respectively (Tables $5-7$ ). These energies increment the expected trends in a remarkably clear manner.

Unsurprisingly, substituents deploy their strongest effects when located at the ortho position as in substrates 1. Under these circumstances, fluorine turns out again to be the most powerful activator, followed by the other halogen atoms, the trifluoromethyl and the methoxy groups. The bulky trimethylsilyl group totally screens the neighboring sites against base attack.

$$
\text { ortho: } \mathrm{F}>\mathrm{Cl} \gtrsim \mathrm{Br} \gtrsim \mathrm{I}>\mathrm{CF}_{3} \gtrsim \mathrm{OMe} \gg \mathrm{H} \gg \mathrm{SiMe}_{3}
$$

Table 5. Substituent effects on the reaction between substrates 1-4 and LIS in THF at $-75{ }^{\circ} \mathrm{C}$ : differences in the free activation energies relative to the unsubstituted substrates ${ }^{a}$

\begin{tabular}{lcccc}
\hline $\mathrm{X}$ & meta-X & meta-X & ortho-X \\
& 0.00 & 0.00 & 0.00 & 0.00 \\
$\mathrm{H}$ & - & -0.29 & -0.12 & -0.038 \\
$\mathrm{SiMe}_{3}$ & +1.9 & +1.2 & +0.91 & -0.29 \\
$\mathrm{~F}$ & +1.8 & +1.5 & +1.3 & +0.17 \\
$\mathrm{Cl}$ & +1.5 & +1.6 & +1.4 & - \\
$\mathrm{CF}_{3}$ & & & & $\mathbf{3}$
\end{tabular}

${ }^{\mathrm{a}} \Delta \Delta \mathrm{G}^{\ddagger}\left(\mathrm{kcal} \mathrm{mol}^{-1}\right)=0.9067 \times\left(\lg k_{\mathrm{X}}-\lg k_{\mathrm{H}}\right)$.

Table 6. Substituent effects on the reaction between substrates 1-4 and LiTMP in THF at -75 ${ }^{\circ} \mathrm{C}$ : differences in the free activation energies relative to the unsubstituted substrates ${ }^{\mathrm{a}}$

\begin{tabular}{lcccc}
\hline $\mathrm{X}$ & ortho-X & meta-X & meta-X \\
& 0.00 & 0.00 & 0.00 & 0.00 \\
$\mathrm{H}$ & - & -0.37 & -0.44 & -0.011 \\
$\mathrm{SiMe}_{3}$ & +2.0 & +0.42 & +0.44 & -0.14 \\
$\mathrm{OMe}$ & +2.9 & +1.5 & +1.5 & +0.013 \\
$\mathrm{~F}$ & +2.8 & +1.8 & +2.0 & +0.46 \\
$\mathrm{Cl}$ & +2.6 & +1.9 & +2.3 & +0.44 \\
$\mathrm{Br}$ & +2.6 & +1.9 & +2.3 & +0.39 \\
$\mathrm{I}$ & +2.0 & +2.1 & +1.9 & - \\
$\mathrm{CF}_{3}$ & +2.9 & & \\
\hline
\end{tabular}

${ }^{\mathrm{a}} \Delta \Delta \mathrm{G}^{\ddagger}\left(\mathrm{kcal} \mathrm{mol}{ }^{-1}\right)=0.9067 \times\left(\lg k_{\mathrm{X}}-\lg k_{\mathrm{H}}\right)$. 
Table 7. Substituent effects on the equilibrium positions between the LiTMP-mediated metalation products of substrates 1-4 in THF at $-75{ }^{\circ} \mathrm{C}$ : differences in the thermodynamic stabilities relative to the unsubstituted metalated substrates ${ }^{\mathrm{a}}$

\begin{tabular}{lcccc}
\hline $\mathrm{X}$ & ortho-X & meta-X & meta-X & 2ara-X \\
& 0.00 & 0.00 & 0.00 & 0.00 \\
$\mathrm{H}$ & - & -0.60 & -0.50 & -0.19 \\
$\mathrm{SiMe}$ & +1.8 & +0.46 & +0.29 & -0.48 \\
$\mathrm{OMe}$ & +7.4 & +2.2 & +1.9 & +0.48 \\
$\mathrm{~F}$ & +6.4 & +2.5 & +2.7 & +1.3 \\
$\mathrm{Cl}$ & +6.4 & +2.7 & +3.0 & +1.2 \\
$\mathrm{Br}$ & +5.5 & +2.4 & +2.9 & +1.1 \\
$\mathrm{I}$ & +5.2 & +3.2 & +3.2 & - \\
$\mathrm{CF}_{3}$ & & & &
\end{tabular}

${ }^{\mathrm{a}} \Delta \mathrm{G}^{\circ}\left(\mathrm{kcal} \mathrm{mol}^{-1}\right)=0.9067 \times\left(\lg K_{\mathrm{X}}-\lg K_{\mathrm{H}}\right)$.

The substituents occupy meta positions with respect to the deprotonation sites in substrates 2 and 3. Similar substituent effects were observed in both series. In some cases (e.g. with $X=$ $\mathrm{SiMe}_{3}$ ), buttressing effects ${ }^{34-41}$ (steric effects exerted by the $\mathrm{X}$ group through the fluoro group) ${ }^{42}$ may compromise the reactivity of the 1,2-isomers $\mathbf{2}$. More importantly, the acidifying effect of substituents at meta positions is no longer the same as at ortho positions. Trifluoromethyl, which appears to cause steric hindrance in its immediate vicinity, turns out to be the most potent activator if more remote from the metalation center. The heavier halogens prove this time to be more effective that fluorine. This can explain why, whereas 1,3-dibromobenzene wins in a competitive reaction with fluorobenzene using LiTMP, ${ }^{31}$ 2,4-dibromo-1-fluorobenzene is deprotonated next to fluorine. ${ }^{43}$ Methoxy maintains only a small rate and stability enhancing effect whereas trimethylsilyl retards and destabilizes slightly.

$$
\text { meta: } \mathrm{CF}_{3}>\mathrm{Br} \gtrsim \mathrm{I}>\mathrm{Cl}>\mathrm{F}>\mathrm{OMe}>\mathrm{H}>\mathrm{SiMe}_{3}
$$

It is already known that trifluoromethyl loses little of its former power, when moved from meta to para. ${ }^{44}$ In contrast, the heavy halogen atoms chlorine, bromine and iodine are far less effective when accommodated at para rather than at meta positions. As previously observed in the case of methoxy using LIS $^{45}$ fluorine and methoxy may even retard metalation. Trimethylsilyl was found to diminish rates and stabilities when located para to the deprotonation site.

$$
\text { para: } \mathrm{CF}_{3} \gg \mathrm{Cl} \gtrsim \mathrm{Br} \gtrsim \mathrm{I} \gg \mathrm{H} \sim \mathrm{F}>\mathrm{SiMe}_{3}>\mathrm{OMe}
$$


The impact of the substituents increases considerably when one moves from LIS- to LiTMPpromoted reactions and, all the more, to LiTMP-mediated equilibrations. For example, orthofluorine enhances the kinetic or thermodynamic proton mobility by approximately 2 (Table 5), 3 (Table 6) and 7 (Table 7) powers of ten. This trend was predictable. Aryllithium ground states should mirror stabilizing substituent effects more pronouncedly than transition states which represent organometallic intermediates merely in statu nascendi or, in other words, as chimeras. Being a weaker base than LIS, LiTMP will develop a more aryllithium-like and hence more substituent-sensitive transition state. ${ }^{46}$

A closer look at the numbers reveals some conspicuous 'anomalies'. Unlike all other substituents, ortho-methoxy is more powerful under kinetic rather than thermodynamic conditions (Table $6 \mathrm{vs}$. 7). This means it entertains at the ground state, if any a smaller coordinative interaction than at the transition state. ${ }^{4,30,47,48}$ Conversely, ortho-trifluoromethyl displays at the level of rates, due to the steric hindrance it exerts, only a shadow of the stabilizing potential it confers on equilibria.

We were fortunate to identify conditions allowing us to assess the rates of LiTMP-promoted metalation by in situ trapping and the thermodynamic stability (i.e. basicity) of the species thus generated by LiTMP/HTMP-mediated equilibration. However, the latter approach is not always applicable and, therefore, must be verified from case to case. For example, 2,4bis(trifluoromethyl)phenyllithium does not isomerize to its less basic 2,6-isomer in the presence of HTMP. ${ }^{44}$

\section{Conclusions}

Thus, the effects of different substituents on the rate of fluorobenzene deprotolithiation were studied. As expected, the substituent at the ortho position displays the strongest effet, and the champion is fluorine. Nevertheless, the effect of the different substituents at the meta and para positions are specific, e.g. with trifluoromethyl, chlorine, bromine and iodine exhibiting a long range acidifying effect. These established data are useful for predictions.

\section{Experimental Section}

General: For laboratory routines, see previous publications from this laboratory. ${ }^{22,34-41}$

\section{Starting materials and products}

(a) Starting materials: Fluorobenzene, difluorobenzenes, 1,3,5-trifluorobenzene, chlorofluorobenzenes, 1-chloro-3,5-difluorobenzene, bromofluorobenzenes, 1-bromo-3,5difluorobenzene, fluoroiodobenzenes, fluoroanisoles, 3,5-difluoroanisole and fluorobenzotrifluorides are commercially available. (4-Fluorophenyl)trimethylsilane, ${ }^{41}(3,5-$ 
difluorophenyl)trimethylsilane ${ }^{41}$ and 1,3-difluoro-5-iodobenzene (bp 63-64 ${ }^{\circ} \mathrm{C}$, lit. ${ }^{49}$ 58-60 ${ }^{\circ} \mathrm{C}$ ) were prepared by bromine-magnesium exchange of the corresponding bromofluorobenzene or bromodifluorobenzene, as previously described for the synthesis of 3,5-difluoro-4(trimethylsilyl)benzoic acid. ${ }^{37}$ (2-Fluorophenyl)trimethylsilane ${ }^{41}$ was prepared by deprotonation of fluorobenzene using LIS in THF at $-75^{\circ} \mathrm{C}$, as previously described for the synthesis of (2fluorophenyl)triethylsilane. ${ }^{50}$

(b) Known products: 2-Fluorobenzoic acid, ${ }^{51}$ (2-fluorophenyl)trimethylsilane, ${ }^{41}$ 2-fluoro-6methoxybenzoic acid, ${ }^{30}$ 2,6-difluorobenzoic acid, ${ }^{52}$ (2,6-difluorophenyl)trimethylsilane, ${ }^{53}$ 2chloro-6-fluorobenzoic acid, ${ }^{54}$ (2-chloro-6-fluorophenyl)trimethylsilane, ${ }^{53}$ 2-bromo-6fluorobenzoic acid, ${ }^{55}$ 2-fluoro-6-iodobenzoic acid, ${ }^{56}$ 2-fluoro-6-(trifluoromethyl)benzoic acid, ${ }^{57}$ 2-fluoro-3-(trimethylsilyl)benzoic acid $^{41}$ (2-fluoro-1,3-phenylene)bis(trimethylsilane), ${ }^{58}$ 2fluoro-3-methoxybenzoic $\quad$ acid $^{59}$ 2,3-difluorobenzoic $\quad$ acid $^{52} \quad(2,3-$ difluorophenyl)trimethylsilane, ${ }^{41} \quad$ 3-chloro-2-fluorobenzoic $\quad$ acid, ${ }^{60}$ (3-chloro-2fluorophenyl)trimethylsilane, ${ }^{61} \quad$ 3-bromo-2-fluorobenzoic $\quad$ acid, ${ }^{60}$ (3-bromo-2fluorophenyl)trimethylsilane, ${ }^{61} \quad$ 2-fluoro-3-(trifluoromethyl)benzoic $\quad$ acid, ${ }^{57} \quad$ 2-fluoro-5(trimethylsilyl)benzoic acid, ${ }^{41}$ 2-fluoro-5-methoxybenzoic acid, ${ }^{59}$ (2-fluoro-5methoxyphenyl)trimethylsilane, ${ }^{62} \quad 2,5$-difluorobenzoic $\quad$ acid, ${ }^{63} \quad(2,5-$ difluorophenyl)trimethylsilane, ${ }^{41}$ 5-chloro-2-fluorobenzoic acid, $^{64}$ 5-bromo-2-fluorobenzoic acid, $^{65}$ 2-fluoro-5-(trifluoromethyl)benzoic acid, ${ }^{66}$ 2,6-difluorobenzoic acid, $^{52} \quad(2,6-$ difluorophenyl)trimethylsilane, ${ }^{53}$ 2,6-difluoro-4-(trimethylsilyl)benzoic acid, ${ }^{41}$ 2,6-difluoro-4methoxybenzoic acid, ${ }^{67}$ 2,4,6-trifluorobenzoic acid $^{68} \quad\left(\mathrm{mp} \quad 142-144 \quad{ }^{\circ} \mathrm{C}\right), \quad(2,4,6-$ trifluorophenyl)trimethylsilane, ${ }^{41}$ (4-chloro-2,6-difluorophenyl)trimethylsilane, ${ }^{69}$ (4-bromo-2,6difluorophenyl)trimethylsilane ${ }^{37}$ and 4-bromo-2,6-difluorobenzoic acid ${ }^{43}$ have been previously described.

\section{c) New products:}

(2-Bromo-6-fluorophenyl)trimethylsilane: bp $39-41{ }^{\circ} \mathrm{C} / 0.3 \mathrm{~mm} \mathrm{Hg} ; \mathrm{n}_{\mathrm{D}}^{20} 1.5245 ; \mathrm{d} 1.33 ;{ }^{1} \mathrm{H}$ NMR $\left(\mathrm{CDCl}_{3}\right) \delta 7.42(1 \mathrm{H}, \mathrm{dd}, J 8.1$ and $0.9 \mathrm{~Hz}), 7.28(1 \mathrm{H}, \mathrm{td}, J 8.3$ and $6.2 \mathrm{~Hz}), 7.04(1 \mathrm{H}, \mathrm{ddd}, J$ 9.7, 8.6 and $0.9 \mathrm{~Hz}), 0.46(9 \mathrm{H}, \mathrm{d}, J 2.5 \mathrm{~Hz}) ;{ }^{13} \mathrm{C} \mathrm{NMR}\left(\mathrm{CDCl}_{3}\right) \delta 167.1(\mathrm{~d}, J 246 \mathrm{~Hz}), 131.7(\mathrm{~d}, J$ $9.6 \mathrm{~Hz}), 130.1$ (d, $J 13 \mathrm{~Hz}), 129.4,127.4$ (d, $J 31 \mathrm{~Hz}), 114.2$ (d, $J 28 \mathrm{~Hz}), 1.3$ (d, J 4.0 Hz); microanalysis calc. for $\mathrm{C}_{9} \mathrm{H}_{12} \mathrm{BrFSi}$ (247.19) $\mathrm{C} 43.73, \mathrm{H} 4.89$; found $\mathrm{C} 43.65, \mathrm{H} 4.98 \%$.

(2-Fluoro-6-iodophenyl)trimethylsilane: bp $46-48{ }^{\circ} \mathrm{C} / 0.3 \mathrm{~mm} \mathrm{Hg} ;{ }^{1} \mathrm{H} \mathrm{NMR}\left(\mathrm{CDCl}_{3}\right) \delta 7.81$ $(1 \mathrm{H}, \mathrm{m}), 7.08(2 \mathrm{H}, \mathrm{m}), 0.49(9 \mathrm{H}, \mathrm{d}, J 2.8 \mathrm{~Hz})$; microanalysis calc. for $\mathrm{C}_{9} \mathrm{H}_{12} \mathrm{FISi}$ (294.18) C 36.75, H 4.11; found C 36.79, H 3.83\%.

2-Fluoro-3-iodobenzoic acid: $\mathrm{mp} 174-176{ }^{\circ} \mathrm{C} ;{ }^{1} \mathrm{H} \mathrm{NMR}\left(\mathrm{CDCl}_{3}\right) \delta 8.16(1 \mathrm{H}, \mathrm{d}, J 7.9 \mathrm{~Hz}), 8.14$ $(1 \mathrm{H}, \mathrm{d}, J 8.0 \mathrm{~Hz}), 7.15(1 \mathrm{H}, \mathrm{t}, J 8.0 \mathrm{~Hz}) ;{ }^{13} \mathrm{C} \mathrm{NMR}\left(\mathrm{CDCl}_{3}\right) \delta 165.7(\mathrm{~d}, J 4.0 \mathrm{~Hz}), 160.7(\mathrm{~d}, J 259$ Hz), 143.7, 132.6, 125.5 (d, J 4.0 Hz), 119.8 (d, J 13 Hz), 83.2 (d, $J 27 \mathrm{~Hz})$; microanalysis calc. for $\mathrm{C}_{7} \mathrm{H}_{4} \mathrm{FIO}_{2}(266.01) \mathrm{C} 31.61, \mathrm{H} 1.52$; found C 31.59, $\mathrm{H} 1.87 \%$. 
(2-Fluoro-3-iodophenyl)trimethylsilane: bp $82-84{ }^{\circ} \mathrm{C} / 2 \mathrm{~mm} \mathrm{Hg} ; \mathrm{n}_{\mathrm{D}}^{20} 1.5532$; $\mathrm{d} 1.45 ;{ }^{1} \mathrm{H} \mathrm{NMR}$ $\left(\mathrm{CDCl}_{3}\right) \delta 7.89(1 \mathrm{H}$, ddd, $J$ 8.2, 6.8 and $1.7 \mathrm{~Hz}), 7.47(1 \mathrm{H}, \mathrm{ddd}, J 7.0,5.2$ and $1.7 \mathrm{~Hz}), 7.00(1 \mathrm{H}$, t, $J 7.6 \mathrm{~Hz}), 0.32(9 \mathrm{H}, \mathrm{d}, J 1.1 \mathrm{~Hz}) ;{ }^{13} \mathrm{C} \mathrm{NMR}\left(\mathrm{CDCl}_{3}\right) \delta 165.3(\mathrm{~d}, J 239 \mathrm{~Hz}), 140.6,131.5(\mathrm{~d}, J$ $10 \mathrm{~Hz}), 127.4(\mathrm{~d}, J 34 \mathrm{~Hz}), 125.6,81.6(\mathrm{~d}, J 31 \mathrm{~Hz}),-1.2$; microanalysis calc. for $\mathrm{C}_{9} \mathrm{H}_{12} \mathrm{FISi}$ (294.18) C 36.75, H 4.11; found C 36.95, H 4.08\%.

(5-Chloro-2-fluorophenyl)trimethylsilane: bp 59-61 ${ }^{\circ} \mathrm{C} / 2 \mathrm{~mm} \mathrm{Hg} ; \mathrm{n}_{\mathrm{D}}^{20} 1.4951 ; \mathrm{d} 1.09 ;{ }^{1} \mathrm{H}$ NMR $\left(\mathrm{CDCl}_{3}\right) \delta 7.42(2 \mathrm{H}, \mathrm{m}), 7.04(1 \mathrm{H}, \mathrm{t}, J 8.2 \mathrm{~Hz}), 0.32(9 \mathrm{H}, \mathrm{d}, J 0.9 \mathrm{~Hz}) ;{ }^{13} \mathrm{C} \mathrm{NMR}\left(\mathrm{CDCl}_{3}\right) \delta$ 165.7 (d, $J 241 \mathrm{~Hz}), 134.6$ (d, J $12 \mathrm{~Hz}), 130.9$ (d, $J 8.9 \mathrm{~Hz}), 129.1,128.7$ (d, $J 33 \mathrm{~Hz}), 116.2$ (d, $J$ $29 \mathrm{~Hz}$ ), -1.2; microanalysis calc. for $\mathrm{C}_{9} \mathrm{H}_{12} \mathrm{ClFSi}$ (202.73) C 53.32, H 5.97; found C 53.28, H $5.91 \%$.

(5-Bromo-2-fluorophenyl)trimethylsilane: bp $72-74{ }^{\circ} \mathrm{C} / 3 \mathrm{~mm} \mathrm{Hg} ; \mathrm{n}_{\mathrm{D}}^{20} 1.5145 ; \mathrm{d} 1.28 ;{ }^{1} \mathrm{H}$ NMR $\left(\mathrm{CDCl}_{3}\right) \delta 7.57(2 \mathrm{H}, \mathrm{m}), 7.00(1 \mathrm{H}, \mathrm{t}, J 8.3 \mathrm{~Hz}), 0.32(9 \mathrm{H}, \mathrm{d}, J 0.9 \mathrm{~Hz}) ;{ }^{13} \mathrm{C} \mathrm{NMR}\left(\mathrm{CDCl}_{3}\right) \delta$ 166.2 (d, $J 242 \mathrm{~Hz}), 137.5$ (d, $J 12 \mathrm{~Hz}), 133.9$ (d, $J 8.9 \mathrm{~Hz}), 129.4$ (d, $J 32 \mathrm{~Hz}), 116.9,116.7$ (d, $J$ $28 \mathrm{~Hz}$ ), -1.2; microanalysis calc. for $\mathrm{C}_{9} \mathrm{H}_{12} \mathrm{BrFSi}(247.19) \mathrm{C} 43.73, \mathrm{H} 4.89$; found $\mathrm{C} 43.79, \mathrm{H}$ $5.03 \%$.

2-Fluoro-5-iodobenzoic acid: $\mathrm{mp} 162-164{ }^{\circ} \mathrm{C} ;{ }^{1} \mathrm{H}$ NMR $\left(\mathrm{CDCl}_{3}\right) \delta 8.47(1 \mathrm{H}$, dd, $J 6.9$ and 2.4 $\mathrm{Hz}), 8.01(1 \mathrm{H}$, ddd, $J$ 6.9, 4.5 and $2.4 \mathrm{~Hz}), 7.08(1 \mathrm{H}, \mathrm{dd}, J 10.5$ and $8.9 \mathrm{~Hz})$; microanalysis calc. for $\mathrm{C}_{7} \mathrm{H}_{4} \mathrm{FIO}_{2}$ (266.01) C 31.61, $\mathrm{H} 1.52$; found $\mathrm{C} \mathrm{31.76,} \mathrm{H} 1.62 \%$.

(2-Fluoro-5-iodophenyl)trimethylsilane: bp $54-56{ }^{\circ} \mathrm{C} / 0.2 \mathrm{~mm} \mathrm{Hg} ; \mathrm{n}_{\mathrm{D}}^{20} 1.5466 ;{ }^{1} \mathrm{H} \mathrm{NMR}$ $\left(\mathrm{CDCl}_{3}\right) \delta 7.60(2 \mathrm{H}, \mathrm{m}), 6.75(1 \mathrm{H}, \mathrm{td}, J 8.3$ and $0.7 \mathrm{~Hz}), 0.32(9 \mathrm{H}, \mathrm{d}, J 0.9 \mathrm{~Hz}) ;{ }^{13} \mathrm{C}$ NMR $\left(\mathrm{CDCl}_{3}\right) \delta 167.5(\mathrm{~d}, J 241 \mathrm{~Hz}), 143.6(\mathrm{~d}, J 11 \mathrm{~Hz}), 139.9(\mathrm{~d}, J 8.8 \mathrm{~Hz}), 130.1(\mathrm{~d}, J 33 \mathrm{~Hz}), 117.2$ (d, $J 27 \mathrm{~Hz}), 88.0$ (d, $J 2.4 \mathrm{~Hz}$ ), -1.2; microanalysis calc. for $\mathrm{C}_{9} \mathrm{H}_{12} \mathrm{FISi}(294.18) \mathrm{C} 36.75, \mathrm{H} 4.11$; found $\mathrm{C} 36.82, \mathrm{H} 4.15 \%$.

4-Chloro-2,6-difluorobenzoic acid: $\mathrm{mp} 161-163{ }^{\circ} \mathrm{C} ;{ }^{1} \mathrm{H} \mathrm{NMR}\left(\mathrm{CDCl}_{3}\right) \delta 7.12(2 \mathrm{H}, \mathrm{d}, J 7.6 \mathrm{~Hz})$; ${ }^{13} \mathrm{C} \mathrm{NMR}\left(\mathrm{CDCl}_{3}\right) \delta 162.8,160.9(\mathrm{dd}, J 259$ and $8.0 \mathrm{~Hz}), 137.9(\mathrm{~m}), 113.2(\mathrm{~d}, J 26 \mathrm{~Hz}), 110.6$; microanalysis calc. for $\mathrm{C}_{7} \mathrm{H}_{3} \mathrm{ClF}_{2} \mathrm{O}_{2}$ (192.55) $\mathrm{C} 43.67, \mathrm{H} \mathrm{1.57}$; found $\mathrm{C} 43.62, \mathrm{H} 1.61 \%$.

2,6-Difluoro-4-iodobenzoic acid: mp 179-181 ${ }^{\circ} \mathrm{C}$; ${ }^{1} \mathrm{H}$ NMR $\left(\mathrm{CDCl}_{3}\right) \delta 7.47(2 \mathrm{H}, \mathrm{d}, J 7.1 \mathrm{~Hz})$; ${ }^{13} \mathrm{C} \mathrm{NMR}\left(\mathrm{CDCl}_{3}\right) \delta 163.4,160.3(\mathrm{dd}, J 262$ and $7.2 \mathrm{~Hz}), 121.8(\mathrm{~d}, J 26 \mathrm{~Hz}), 111.7(\mathrm{~m}), 95.7(\mathrm{~d}$, $J 10 \mathrm{~Hz}$ ); microanalysis calc. for $\mathrm{C}_{7} \mathrm{H}_{3} \mathrm{~F}_{2} \mathrm{IO}_{2}$ (284.00) C 29.60, H 1.06; found C 29.62, H 1.12\%.

Relative rates and equilibria: The working procedure applied when LIS served as the metalating reagent was essentially the same as previously described. ${ }^{22}$ The two other protocols were newly designed.

(a) Competitive reactions with LIS followed by carboxylation: A pair of two substrates (about $10 \mathrm{mmol}$ each) and a known amount (about $2 \mathrm{mmol}$ ) of the "internal standard" decane were dissolved in THF $(20 \mathrm{~mL})$. A tiny fraction was withdrawn for gas chromatographic analysis $\left(2 \mathrm{~m}, 5 \% \mathrm{C}-20 \mathrm{M}\right.$ and $2 \mathrm{~m}, 5 \mathrm{Ap}-\mathrm{L}$, same conditions for both columns: $30{ }^{\circ} \mathrm{C}(10 \mathrm{~min}) \rightarrow 220{ }^{\circ} \mathrm{C}$ (heating rate $\left.10{ }^{\circ} \mathrm{C} \mathrm{min}^{-1}\right)$ ). A precooled $\left(-100{ }^{\circ} \mathrm{C}\right)$ solution of LIS $(10 \mathrm{mmol})$ in cyclohexane 
$(7.0 \mathrm{~mL})$ was added under vigorous stirring to the mixture kept in a dry ice/acetone bath. After $15 \mathrm{~min}$ at $-75{ }^{\circ} \mathrm{C}$, the mixture was poured on an excess of freshly crushed dry ice. Once the residual carbon dioxide had been evaporated at $+25{ }^{\circ} \mathrm{C}$, the residue was dissolved in a $1.0 \mathrm{M}$ aqueous solution $(20 \mathrm{~mL})$ of sodium hydroxide. The unconsumed substrates and the standard decane were extracted with diethyl ether $(3 \times 15 \mathrm{~mL})$ and the relative concentrations were determined by gas chromatography (see above). The relative rates were calculated using the familiar logarithmic expression..$^{70,71}$ As a cross-check, the aqueous phase was acidified to $\mathrm{pH} 1$ and extracted with diethyl ether $(3 \times 15 \mathrm{~mL})$. The combined organic layers were concentrated before being treated with ethereal diazomethane until persistence of the yellow color. The methyl esters were again identified by gas chromatography and quantified using methyl benzoate as a separately calibrated internal standard. The amounts of methyl esters thus determined were found in all cases to equal approximately the difference between the initial and recovered substrates.

(b) Competitive reactions with LiTMP in the presence of chlorotrimethylsilane: A precooled $\left(-100{ }^{\circ} \mathrm{C}\right)$ solution of LiTMP (obtained by adding a solution of butyllithium (10 mmol) in hexanes $(7.0 \mathrm{~mL})$ to a solution of 2,2,6,6-tetramethylpiperidine $(1.7 \mathrm{~mL}, 1.4 \mathrm{~g}, 10$ mmol) in THF $(5 \mathrm{~mL})$ at $\left.0{ }^{\circ} \mathrm{C}\right)$ was added under vigorous stirring to a THF $(15 \mathrm{~mL})$ solution containing a known amount of decane (as an "internal standard", approx. 5 mmol), chlorotrimethylsilane $(13 \mathrm{~mL}, 11 \mathrm{~g}, 0.10 \mathrm{~mol}$ ) and the pair of two substrates (approx. $10 \mathrm{mmol}$ each) kept in a dry ice/acetone bath. After being stored for $2 \mathrm{~h}$ at $-75^{\circ} \mathrm{C}$, the mixture was acidified with $10 \%$ hydrochloric acid $(20 \mathrm{~mL})$ and extracted with diethyl ether $(3 \times 20 \mathrm{~mL})$. The concentrations of the unconsumed substrates, required for the calculation ${ }^{70,71}$ of the rate ratios, and the concentrations of the silanes formed, used as a control, were again assessed by gas chromatography (conditions as specified in the preceding paragraph).

(c) Competitive reactions with LiTMP under equilibration conditions: A mixture was prepared as described in paragraph b), but chlorotrimethylsilane was omitted. After $2 \mathrm{~h}$ at -75 ${ }^{\circ} \mathrm{C}$, it was poured onto an excess of freshly crushed dry ice and worked up as specified in paragraph a).

Control reactions: The time dependence of some key reactions was examined. Typical aryllithiums were found to react with chlorotrimethylsilane almost instantaneously. The transmetalation between 2,5-difluorophenyllithium and 1-chloro-4-fluorobenzene or 2-fluoro-5chlorophenyllithium and 1,4-difluorobenzene proceeded with extreme sluggishness in THF at $75{ }^{\circ} \mathrm{C}$ whereas acid-base equilibrium was attained between the same four components in $45 \mathrm{~min}$ when 2,2,6,6-tetramethylpiperidine was present.

(a) Reactivity of aryllithiums toward chlorotrimethylsilane: To a solution of fluorobenzene (1a, $0.94 \mathrm{~mL}, 0.96 \mathrm{~g}, 10 \mathrm{mmol})$ and decane $(1.5 \mathrm{~mL}, 1.1 \mathrm{~g}, 7.5 \mathrm{mmol})$ in THF (20 mL) cooled in a dry ice/acetone bath, was added a solution of LIS $(10 \mathrm{mmol})$ in cyclohexane $(7.0 \mathrm{~mL})$. After 2 
$\mathrm{h}$ at $-75^{\circ} \mathrm{C}$, chlorotrimethylsilane $(1.3 \mathrm{~mL}, 1.1 \mathrm{~g}, 10 \mathrm{mmol})$ was added under vigorous stirring and, $30 \mathrm{~s}$ later, $10 \%$ hydrochloric acid $(10 \mathrm{~mL})$. The aqueous layer was saturated with sodium chloride. According to gas chromatography (same conditions as above), the organic phase contained $>90 \%$ of (2-fluorophenyl)trimethylsilane (2b) and $<10 \%$ of fluorobenzene. Replacement of fluorobenzene by 3-fluorobenzotrifluoride (3c) or 1,3,5-trifluorobenzene (4d) gave similar results.

(b) Reaction between fluoroarenes and 2-fluoroaryllithiums: 1,4-Difluorobenzene (1.0 mL, $1.1 \mathrm{~g}, 10 \mathrm{mmol})$ was treated with LIS $(10 \mathrm{mmol})$ as described in the preceding paragraph. After 2 $\mathrm{h}$ at $-75^{\circ} \mathrm{C}, 1$-chloro-4-fluorobenzene $(1.1 \mathrm{~mL}, 1.3 \mathrm{~g}, 10 \mathrm{mmol})$ was added. In intervals samples $(1.0 \mathrm{~mL})$ were withdrawn and poured in a test tube containing an excess of freshly crushed dry ice. When the mixture had reached $+25{ }^{\circ} \mathrm{C}$, the proportions of remaining 1,4-difluorobenzene and 1-chloro-4-fluorobenzene was probed by gas chromatography (see above) and corrected by calibration factors (Table 8, left-hand column). A second reaction was started by treating 1chloro-4-fluorobenzene with LIS and adding 1,4-difluorobenzene later. Samples were withdrawn and analyzed as before (Table 8, right-hand column).

Table 8. Reaction between 2,5-difluorophenyllithium and 1-chloro-4-fluorobenzene and 5chloro-2-fluorophenyllithium and 1,4-difluorobenzene as a function of time: ratios of residual fluoroarenes (after carboxylation of the fluoroaryllithiums)

\begin{tabular}{ccc}
\hline Time $[\mathrm{h}]$ & 1,4-difluorobenzene series $\mathrm{A}^{\mathrm{a}}$ & vs. 1-chloro-4-fluorobenzene series $\mathrm{B}^{\mathrm{b}}$ \\
\hline 0 & $0: 100$ & $100: 0$ \\
2 & $9: 91$ & $93: 7$ \\
20 & $26: 74$ & $87: 13$ \\
200 & $59: 41$ & $77: 23$ \\
$\infty^{\mathrm{c}}$ & $72: 28$ & $72: 28$ \\
\hline
\end{tabular}

${ }^{\mathrm{a}}$ Starting from 2,5-difluorophenyllithium and 1-chloro-4-fluorobenzene. ${ }^{\mathrm{b}}$ Starting from 5chloro-2-fluorophenyllithium and 1,4-difluorobenzene. ${ }^{\mathrm{c}}$ Equilibrium attained after estimated $2000 \mathrm{~h}$.

(c) Fluoroarene/2-fluoroaryllithium equilibration in the presence of 2,2,6,6tetramethylpiperidine: A precooled $\left(-100{ }^{\circ} \mathrm{C}\right.$ ) solution of LiTMP (obtained by adding a solution of butyllithium $(10 \mathrm{mmol})$ in hexanes $(7.0 \mathrm{~mL})$ to a solution of $2,2,6,6$ tetramethylpiperidine $(1.7 \mathrm{~mL}, 1.4 \mathrm{~g}, 10 \mathrm{mmol})$ in $\mathrm{THF}(5 \mathrm{~mL})$ at $\left.0{ }^{\circ} \mathrm{C}\right)$ was added to a solution of 1,4-difluorobenzene (1.0 mL, $1.1 \mathrm{~g}, 10 \mathrm{mmol}), 1$-chloro-4-fluorobenzene $(1.1 \mathrm{~mL}, 1.3 \mathrm{~g}, 10$ $\mathrm{mmol}$ ) and decane (as the "internal standard"; $1.5 \mathrm{~mL}, 1.1 \mathrm{~g}, 7.5 \mathrm{mmol}$ ) in THF (15 mL) kept in a dry ice/acetone bath. Samples $(2.0 \mathrm{~mL})$, withdrawn in intervals, were poured on an excess of freshly crushed dry ice. At $+25^{\circ} \mathrm{C}$, the liquid was decanted and the residue washed with diethyl ether $(15 \mathrm{~mL})$. The combined organic layers were filtered and analyzed by gas chromatography 
(see above) to determine the proportions of unconsumed 1,4-difluorobenzene and 1-chloro-4fluorobenzene (Table 9).

Table 9. Reaction between lithium 2,2,6,6-tetramethylpiperidide and a 1:1 mixture of 1,4difluorobenzene and 1-chloro-4-fluorobenzene in THF at $-75^{\circ} \mathrm{C}$ : ratios of residual fluoroarenes (after carboxylation of the fluoroaryllithiums)

\begin{tabular}{lc}
\hline Time $[\mathrm{min}]$ & Ratio $^{\mathrm{a}}$ 1,4-difluorobenzene $v s$. 1-chloro-4-fluorobenzene \\
\hline 0 & $56: 44^{\mathrm{b}}$ \\
0.5 & $57: 43$ \\
5 & $60: 40$ \\
.15 & $64: 36$ \\
25 & $68: 32$ \\
35 & $71: 29$ \\
45 & $73: 27$ \\
60 & $72: 28$ \\
120 & $73: 29$ \\
360 & $72: 28$ \\
\hline
\end{tabular}

${ }^{\mathrm{a}}$ After correction for different response factors using the calibrated standard decane. ${ }^{\mathrm{b}}$ Assessed by the "in situ trapping" method (see Section 2b).

\section{Acknowledgements}

This project was supported by the Schweizerische Nationalfond zur Förderung der wissenschaftlichen Forschung, Bern (grant 20-100'336-02).

\section{References}

¥ Current address: Chimie et Photonique Moléculaires, Institut des Sciences Chimiques de Rennes, UMR 6226, Université de Rennes 1-CNRS, Bâtiment 10A, Case 1003, Campus de Beaulieu, 35042 Rennes, France

1. Wittig, G. Angew. Chem. 1954, 66, 10-17. http://dx.doi.org/10.1002/ange.19540660103

2. Wittig, G. Experientia 1958, 14, 389-395 (Chem. Abstr. 1959, 53, 94225).

3. Wittig, G.; Köbrich, G. Endeavour 1969, 28, 123-128 (Chem. Abstr. 1969, 71, 123080).

4. Schlosser, M. In Organometallics in Synthesis : A Manual; Schlosser M. Ed.; 2nd Ed.; Wiley: Chichester, 2002; pp 1-352, spec. p 47 and 50-72. 
5. O'Sullivan, W. I.; Swamer, F. W.; Humphlett, W. J.; Hauser, C. R. J. Org. Chem. 1961, 26, 2306-2310. http://dx.doi.org/10.1021/jo01351a038

6. DePuy, C. H.; Gronert, S.; Barlow, S. E.; Bierbaum, V. M.; Damrauer, R. J. Am. Chem. Soc. 1989, 111, 1968-1973. http://dx.doi.org/10.1021/ja00188a003

7. Holm, T. J. Organomet. Chem. 1974, 77, 27-30. http://dx.doi.org/10.1016/S0022-328X(00)89416-4

8. Arnett, E. M.; Moe, K. D. J. Am. Chem. Soc. 1991, 113, 7068-7069. http://dx.doi.org/10.1021/ja00018a071

9. Hyla-Kryspin, I.; Grimme, S.; Javor, S.; Masson, E.; Schlosser, M., unpublished results.

10. Wenthold, P. G.; Squires, R. R. J. Mass Spectrom. 1995, 30, 17-24. http://dx.doi.org/10.1002/jms.1190300105

11. Andrade, P. B. M.; Riveros, J. M. J. Mass Spectrom. 1996, 31, 767-770. http://dx.doi.org/10.1002/(SICI)1096-9888(199607)31:7<767::AID-JMS345>3.0.CO;2-Q

12. Büker, H. H.; Nibbering, N. M. M.; Espinosa, D.; Mongin, F.; Schlosser, M. Tetrahedron Lett. 1997, 38, 8519-8522. http://dx.doi.org/10.1016/S0040-4039(97)10303-3

13. Hyla-Kryspin, I.; Grimme, S.; Büker, H. H.; Nibbering, N. M. M.; Cottet, F.; Schlosser, M. Chem. Eur. J. 2005, 11, 1251-1256. http://dx.doi.org/10.1002/chem.200400967

14. Hall, G. E.; Piccolini, R.; Roberts, J. D. J. Am. Chem. Soc. 1955, 77, 4540-4543. http://dx.doi.org/10.1021/ja01622a033

15. Yurygina, E. N.; Alikhanov, P. P.; Izrailevich, E. A.; Manochkina, P. N.; Shatenstein, A. I. Zhur. Fiz. Khim. 1960, 34, 587-593 (Chem. Abstr. 1961, 55, 17548g).

16. Shatenshteîn, A. I. Adv. Phys. Org. Chem. 1963, 1, 155-201, spec. p 187. http://dx.doi.org/10.1016/S0065-3160(08)60278-6

17. Streitwieser, A.; Mares, F. J. Am. Chem. Soc. 1968, 90, 644-648. http://dx.doi.org/10.1021/ja01005a016

18. Streitwieser, A.; Juaristi, E.; Nebenzahl, L. L. in Comprehensive Carbanion Chemistry; Buncel, E.; Durst, T. Eds.; Elsevier: Amsterdam, 1980; pp 323-381.

19. Castagnetti, E.; Schlosser, M. Chem. Eur. J. 2002, 8, 799-804. http://dx.doi.org/10.1002/1521-3765(20020215)8:4<799::AID-CHEM799>3.0.CO;2-6

20. Roberts, J. D.; Curtin, D. Y. J. Am. Chem. Soc. 1946, 68, 1658-1660. http://dx.doi.org/10.1021/ja01212a090

21. Shirley, D. A.; Johnson, F. R.; Hendrix, J. P. J. Organomet. Chem. 1968, 11, 209-216. http://dx.doi.org/10.1016/0022-328X(68)80043-9

22. Schlosser, M.; Marzi, E. Chem. Eur. J. 2005, 11, 3449-3454. http://dx.doi.org/10.1002/chem.200401094 
23. Pierce, O. R.; McBee, E. T.; Judd, G. F. J. Am. Chem. Soc. 1954, 76, 474-478.

http://dx.doi.org/10.1021/ja01631a042

24. Seyferth, D.; Weinstein, R. M. J. Am. Chem. Soc. 1982, 104, 5534-5535. http://dx.doi.org/10.1021/ja00384a065

25. Krizan, T. D.; Martin, J. C. J. Am. Chem. Soc. 1983, 105, 6155-6157. http://dx.doi.org/10.1021/ja00357a034

26. Seebach, D.; Weber, T. Tetrahedron Lett. 1983, 24, 3315-3318. http://dx.doi.org/10.1016/S0040-4039(00)86257-7

27. Gassman, P. G.; O'Reilly, N. J. Tetrahedron Lett. 1985, 26, 5243-5246. http://dx.doi.org/10.1016/S0040-4039(00)95005-6

28. Lipshutz, B. H.; Wood, M. R.; Lindsley, C. W. Tetrahedron Lett. 1995, 36, 4385-4388. http://dx.doi.org/10.1016/0040-4039(95)00856-8

29. Schlosser, M.; Guio, L.; Leroux, F. J. Am. Chem. Soc. 2001, 123, 3822-3823. http://dx.doi.org/10.1021/ja0032733

30. Katsoulos, G.; Takagishi, S.; Schlosser, M. Synlett 1991, 731-732. http://dx.doi.org/10.1055/s-1991-34754

31. Mongin, F.; Schlosser, M., unpublished results.

32. Marzi, E.; Schlosser, M., unpublished results.

33. See aso: Wang, L.; Wang, Y.; Guo, F.; Zheng, Y.; Bhadury, P. N.; Sun, Z. Tetrahedron Lett. 2013, 54, 6053-6056. http://dx.doi.org/10.1016/j.tetlet.2013.08.098

34. Heiss, C.; Marzi, E.; Schlosser, M. Eur. J. Org. Chem. 2003, 4625-4629. http://dx.doi.org/10.1002/ejoc.200300355

35. Gorecka, J.; Heiss, C.; Scopelliti, R.; Schlosser, M. Org. Lett. 2004, 6, 4591-4593. http://dx.doi.org/10.1021/o10480022

36. Heiss, C.; Cottet, F.; Schlosser, M. Eur. J. Org. Chem. 2005, 5236-5241. http://dx.doi.org/10.1002/ejoc.200500553

37. Heiss, C.; Leroux, F.; Schlosser, M. Eur. J. Org. Chem. 2005, 5242-5247. http://dx.doi.org/10.1002/ejoc.200500552

38. Schlosser, M.; Cottet, F.; Heiss, C.; Lefebvre, O.; Marull, M.; Masson, E.; Scopelliti, R. Eur. J. Org. Chem. 2006, 729-734.

http://dx.doi.org/10.1002/ejoc.200500728

39. Schlosser, M.; Heiss, C.; Leroux, F. Eur. J. Org. Chem. 2006, 735-737. http://dx.doi.org/10.1002/ejoc.200500729

40. Schlosser, M.; Heiss, C.; Marzi, E.; Scopelliti, R. Eur. J. Org. Chem. 2006, 4398-4404. http://dx.doi.org/10.1002/ejoc.200600350

41. Heiss, C.; Marzi, E.; Mongin, F.; Schlosser, M. Eur. J. Org. Chem. 2007, 669-675. http://dx.doi.org/10.1002/ejoc.200600589 
42. Decouzon, M.; Ertl, P.; Exner, O.; Gal, J.-F.; Maria, P.-C. J. Am. Chem. Soc. 1993, 115, 12071-12078. http://dx.doi.org/10.1021/ ja00078a052

43. Mongin, F.; Schlosser, M. Tetrahedron Lett. 1996, 37, 6551-6554. http://dx.doi.org/10.1016/0040-4039(96)01398-6

44. Schlosser, M.; Mongin, F.; Porwisiak, J.; Dmowski, W.; Büker, H. H.; Nibbering, N. M. M. Chem. Eur. J. 1998, 4, 1281-1286. http://dx.doi.org/10.1002/(SICI)1521-3765(19980710)4:7<1281::AIDCHEM1281>3.0.CO;2-I

45. Maggi, R.; Schlosser, M. Tetrahedron Lett. 1999, 40, 8797-8800. http://dx.doi.org/10.1016/S0040-4039(99)01872-9

46. Takagishi, S.; Schlosser, M. Synlett 1991, 119-121. http://dx.doi.org/10.1055/s-1991-20650

47. Schlosser, M. in Polare Organometalle: Struktur und Reaktivitât; Springer: Berlin, 1973; pp 142-146.

48. Schlosser, M.; Maccaroni, P.; Marzi, E. Tetrahedron 1998, 54, 2763-2770. http://dx.doi.org/10.1016/S0040-4020(98)83011-1

49. Fletcher, T. L.; Namkung, M. J.; Pan, H.-L.; Wetzel, W. H. J. Org. Chem. 1960, 25, 9961000 . http://dx.doi.org/10.1021/jo01076a034

50. Heiss, C.; Rausis, T.; Schlosser, M. Synthesis 2005, 617-621. http://dx.doi.org/10.1055/s-2005-861787

51. Gilman, H.; Gorsich, R. D. J. Am. Chem. Soc. 1956, 78, 2217-2222. http://dx.doi.org/10.1021/ja01591a053

52. Roe, A. M.; Burton, R. A.; Reavill, D. R. Chem. Commun. 1965, 22, 582a. http://dx.doi.org/10.1039/C1965000582A

53. Benneteau, B.; Rajarison, F.; Dunoguès, J.; Babin, P. Tetrahedron 1993, 49, 10843-10854. http://dx.doi.org/10.1016/S0040-4020(01)80238-6

54. Lock, G. Ber. 1936, 69, 2253-2258. http://dx.doi.org/10.1002/cber.19360691008

55. Dewar, M. J. S.; Grisdale, P. J. J. Org. Chem. 1963, 28, 1759-1762. http://dx.doi.org/10.1021/jo01042a006

56. Witiak, D. T.; Goswami, S.; Milo, G. E. J. Org. Chem. 1988, 53, 345-352. http://dx.doi.org/10.1021/jo00237a023

57. Schlosser, M.; Katsoulos, G.; Takagishi, S. Synlett 1990, 747-748. http://dx.doi.org/10.1055/s-1990-21236

58. Dua, S. S.; Gilman, H. Indian J. Chem. B 1979, 17B, 562-565 (Chem. Abstr. 1980, 93, 185318).

59. Pena, M. A.; Sestelo, J. P.; Sarandeses, L. A. J. Org. Chem. 2007, 72, 1271-1275. http://dx.doi.org/10.1021/jo062148s 
60. Moyroud, J.; Guesnet, J.-L.; Bennetau, B.; Mortier, J. Tetrahedron Lett. 1995, 36, 881-884. http://dx.doi.org/10.1016/0040-4039(94)02385-O

61. Moyroud, J.; Guesnet, J.-L.; Bennetau, B.; Mortier, J. Bull. Soc. Chim. Fr. 1996, 133, 133141 (Chem. Abstr. 1996, 125, 58000).

62. Gilday, J. P.; Negri, J. T.; Widdowson, D. A. Tetrahedron 1989, 45, 4605-4618. http://dx.doi.org/10.1016/S0040-4020(01)89096-7

63. Hopff, H.; Valkanas, G. J. Org. Chem. 1962, 27, 2923-2924. http://dx.doi.org/10.1021/jo01055a507

64. Bergmann, E. D.; Berkovic, S. J. Org. Chem. 1961, 26, 919-923. http://dx.doi.org/10.1021/jo01062a067

65. Sedinkin, S. L.; Rath, N. P.; Bauer, E. B. J. Organomet. Chem. 2008, 693, 3081-3091. http://dx.doi.org/10.1016/j.jorganchem.2008.06.031

66. Tidswell, R. J. E.; Jappy, J.; Davison, B. E. Brit. UK Pat. A GB 2191192, 9 Dec. 1987, Appl. GB 1987-12344, 26 May 1987, UK Pat. B, GB 2191192, 14 Nov. 1990 (Chem. Abstr. 1988, 109, 22639).

67. Alonso-Alija, C.; Michels, M.; Peilstöcker, K.; Schirok, H. Tetrahedron Lett. 2004, 45, 9598.

http://dx.doi.org/10.1016/j.tetlet.2003.10.109

68. Patrick, S. R.; Boogaerts, I. I. F.; Gaillard, S.; Slawin, A. M. Z.; Nolan, S. P. Beilstein J. Org. Chem. 2011, 7, 892-896.

http://dx.doi.org/10.3762/bjoc.7.102

69. Balko, T. W.; Schmitzer, P. R.; Daeuble, J. F.; Yerkes, C. N.; Siddall, T. L.; Yerkes, C. N PCT Int. Appl., WO 2007082098 A2, 19 Jul. 2007 (Chem. Abstr. 2007, 147, 159918).

70. Ingold, C. K.; Shaw, F. R. J. Chem. Soc. 1927, 2918-2926. http://dx.doi.org/10.1039/JR9270002918

71. Schlosser, M.; Ladenberger, V. Chem. Ber. 1967, 100, 3901-3915. http://dx.doi.org/10.1002/cber.19671001211 